\title{
Combining active structural damping and active suspension control in flexible bodied railway vehicles
}

\author{
Xiang Zheng ${ }^{1}$, Argyrios Zolotas ${ }^{2}$, Roger Goodall ${ }^{3}$ \\ 1. Key Lab of Marine Technology and Control Engineering, Scientific Research Academy, Shanghai Maritime University, Shanghai \\ 201306, P. R. China \\ E-mail: xiangzheng@shmtu.edu.cn \\ 2. Intelligent Systems Research Group, School of Engineering, College of Science, University of Lincoln, Lincoln, Lincolnshire LN6 7TS, \\ UK \\ E-mail: azolotas@lincoln.ac.uk \\ 3. Control Systems Group, Department of Electronic and Electrical Engineering, Loughborough University, Loughborough, Leicestershire \\ LE11 3TU, UK \\ E-mail: r.m.goodall@lboro.ac.uk
}

\begin{abstract}
There is a desire to design lightweight railway vehicle bodies for future high speed trains. Previously, suppression of structural vibration of the flexible lightweight vehicle body was attempted via use of active suspensions (conventional actuators) or by structural damping via piezoelectric actuators, with the aim being to improve the ride quality. In a railway vehicle the typical active suspension setup comprises front and rear suspension conventional actuators, while adding more macro-actuator elements to minimise structural vibrations can substantially impact vehicle weight and location considerations. In this paper, we show that piezoelectric actuator control can provide complementary action to active suspensions. Decentralized control is adopted for combined active structural damping and active suspension design via Linear Quadratic Gaussian (LQG) method and modal control with skyhook damping respectively. The side-view model of a flexible-bodied railway vehicle integrated with piezoelectric actuators and appropriate sensor outputs is derived and the placement of the piezoelectric actuators and sensors is addressed via structural norms. It is shown that vibrations of both the flexible modes and rigid modes are suppressed effectively. This suggests that with the combined approach, control by both piezoelectric actuators and suspension actuators could be used in a more efficient way to address vibrations in light railway vehicle bodies.
\end{abstract}

Key Words: Railway vehicles, piezoelectric actuators and sensors, active structural damping, active suspension, LQG, Skyhook damping

\section{Introduction}

Future railway vehicles are tend to be designed lighter in order to achieve higher speed and also to be more costeffective and energy-efficient. However, lightweight vehicle bodies are more flexible with structural vibration easily excited by exogenous disturbances, resulting in increased levels of high frequency vibration. Therefore, suppression of vibration of flexible modes besides the rigid modes of the vehicle body is required in order to improve ride quality levels.

Active/semi-active secondary suspension is the effective conventional approach for vibration control of railway vehicles to improve ride quality. Active suspensions and semiactive suspensions were made more aware of the flexible modes and their effectiveness in reducing the structural vibrations of a vehicle body in [4, 5]. Foo and Goodall [2] placed a third actuator in addition to two secondary suspension actuators at the centre of the vehicle for reducing the effects of the first flexible mode of the vehicle body. However, it was shown that the dynamics of the real actuators (e.g. electro-hydraulic actuators) cause a degradation of ride quality at higher frequencies [12] and the suspension points are near the nodes of the first bending mode, which suggest its limitation in suppressing the flexible modes.

Structural damping was applied via the smart structure concept of integrating piezoelectric actuators into the flexible structure. Passive structural damping was designed via shunted piezoelectric elements by electric circuits to dissipate the vibration energy of vehicle body $[7,16]$. Kamada et al. [9] used piezoelectric stack transducers in shunt damping for vibration suppression of a railway vehicle. Active structural damping with piezoelectric actuators was applied by Kamada et al.[8], independent $\mathcal{H}_{\infty}$ controllers were designed for suppressing the first three flexible modes. Schandl et al.[14] attached to the structure piezoelectric stack actuators mounted in consols together with collocated sensor patches, designing state feedback controllers by pole placement with state estimators. In the work by Kozek et al.[11], collocated strain sensors and piezoelectric actuators were used to introduce force/moments pairs into the car body structure and robust $\mathcal{H}_{\infty}$ control was applied. Benatzky et al. [1] designed the controller using the $\mu$-synthesis procedure for structural vibration control of a scaled metro vehicle with piezoelectric stack actuators and piezoelectric patch sensors. A better performance was achieved compared to pole-placement combined with Kalman-filter techniques. Kamada et.al [10] illustrated the effectiveness of combined piezoelectric actuators and linear actuators in suppressing both the rigid and flexible modes with decentralized $\mathcal{H}_{\infty}$ controllers.

Coupling exists between rigid modes and flexible modes [17] and piezoelectric actuators have limitations on the maximum force they can produce, thus it is important to consider suppression of rigid mode and flexible mode vibrations simultaneously. In this paper, we investigate the performance of active structure damping combined with active suspension control. First, the side-view model of a flexible-bodied railway vehicle with piezoelectric actuators and sensors is derived. Then the placement of actuators and sensors is 
done according to $H_{2} / H_{\infty}$ norms. With decentralized control structure, LQG control is applied for active structural damping and modal control with skyhook damping for active suspensions. It is shown that sub controllers work complementary to each other and vibration of both the rigid and flexible modes are suppressed effectively.

\section{Modelling}

\subsection{Side-view model with rigid vehicle body}

The dynamics of a real railway vehicle can be very complex with non-linearities and substantial coupling in certain modes. However, a linear time-invariant model can appropriately represent the real system for the railway suspension$s$ over the required mode of operation. The mathematical model of the system is based on the side-view of a railway vehicle as shown in Figure 1 [2]. It is a simplified model with two wheel sets included instead of four as it is sufficient to address the time delay of input excitations from front to rear bogies.

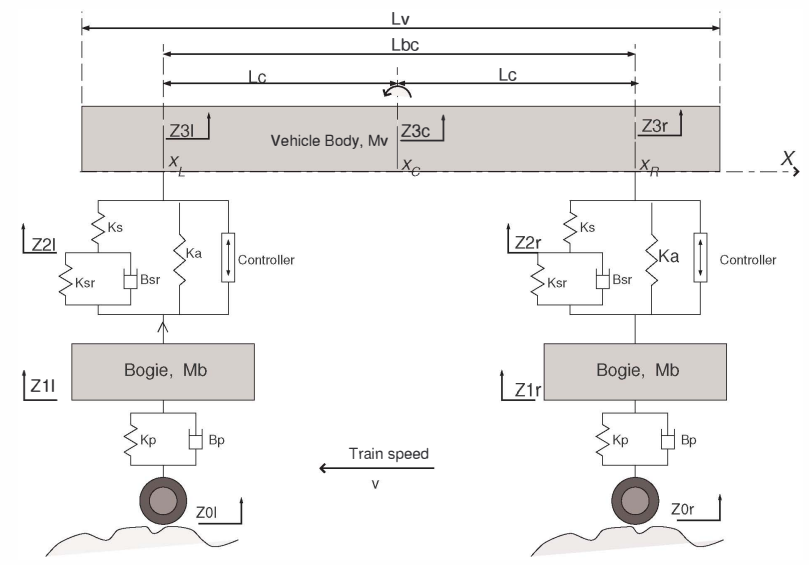

Fig. 1: Sideview model of a typical high speed passenger railway vehicles

The system's degrees of freedom include the vehicle body's bounce and pitch, flexible modes of the vehicle body, bounce modes of the two bogie masses, and two modes associated with the internal dynamics of the air spring. The pitch angle is assumed small so that

$$
\begin{aligned}
& Z_{3 L}=Z_{3 C}+L_{c} \Theta \\
& Z_{3 R}=Z_{3 C}-L_{c} \Theta
\end{aligned}
$$

Lagrangian equations are used to establish the equations of motion and the model is represented in state space form as follows

$$
\dot{\boldsymbol{x}_{\boldsymbol{r}}}=\mathbf{A}_{r} \mathrm{x}+\mathbf{B}_{\mathrm{r}} \boldsymbol{u}+\mathbf{G} \boldsymbol{w}
$$

where $\boldsymbol{u}=\left[\begin{array}{ll}U_{L} & U_{R}\end{array}\right]$ is the control input vector across the secondary suspension, $\boldsymbol{w}=\left[\dot{Z}_{0 L} \dot{Z}_{0 R}\right]$ is the track velocity(disturbance) input vector. $\mathbf{A}_{\mathbf{r}}, \mathbf{B}_{\mathbf{r}}$, and $\mathbf{G}$ are system matrix, force input matrix , and disturbance input matrix respectively. The system state vector $\boldsymbol{x}_{\boldsymbol{r}}$ is set as

$\boldsymbol{x}_{\boldsymbol{r}}=\left[\begin{array}{lllllllll}\dot{Z}_{3 C} & \dot{\theta} & \dot{Z}_{1 L} & \dot{Z}_{1 R} & R_{1} & R_{2} & R_{3} & R_{4} & R_{5}\end{array}\right.$

where

$$
\begin{array}{lll}
R_{1}=Z_{3 L}-Z_{1 L} & R_{2}=Z_{3 R}-Z_{1 R} & R_{3}=Z_{2 L}-Z_{1 L} \\
R_{4}=Z_{2 R}-Z_{1 R} & R_{5}=Z_{1 L}-Z_{0 L} & R_{6}=Z_{1 R}-Z_{0 R}
\end{array}
$$

\subsection{Analytical model of flexible vehicle body with piezo- electric actuators and sensors}

The flexible vehicle body is assumed a free-free EulerBernoulli beam of length, $l$, cross section area, $A_{b}$, mass $M_{b}$, material density, $\rho_{b}$ and flexural rigidity, $E I$, which depends on the Young's modulus of elasticity $E$ and the second moment of area $I$. The flexural vibration of the beam is induced by track disturbances transmitted through the suspensions. Stack piezoelectric actuators as shown in Figure 2 are integrated onto the vehicle body for suppressing the flexible modes. We choose this type of piezoelectric actuator because it is capable of introducing the necessary moments concerning the size, mass and stiffness of the structure[15].

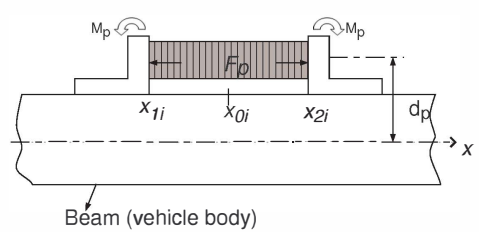

Fig. 2: Piezoelectric actuator

The actuator expands when a voltage $V_{a}$ is applied to it and actuation force $F_{p}$ is generated when this expansion is constrained by mounting the actuator in a mechanical structure. It assumes that the equivalent actuation force is proportional to the applied voltage

$$
F_{p}=A_{p} e_{33} \frac{V_{a}}{h_{S t}}
$$

where $e$ is the piezoelectric coupling matrix, $h_{S t}$ is the thickness of one disk, and $A_{p}$ is the area of cross section of the actuator.

Denote $z(x, t)$ the transverse deflection at point $x$ of the beam and time $t$. The governing partial differential equations (PDEs) that describe the dynamics of the structure of the beam, assuming the beam is one-dimension only and has zero damping, is given as follows [6].

$$
\begin{aligned}
E I \frac{\partial^{4} z(x, t)}{\partial x^{4}} & +\rho_{b} A_{b} \frac{\partial^{2} z(x, t)}{\partial t^{2}}=\sum_{i=1}^{n_{p}} \frac{\partial^{2} M_{p x}(x, t)}{\partial x^{2}} \\
& +F_{x_{L}}(t) \delta\left(x-l_{1}\right)+F_{x_{R}}(t) \delta\left(x-l_{2} \gamma 7\right)
\end{aligned}
$$

where $F_{x_{L}}$ and $F_{x_{F}}$ are the forces acting at the left and right suspension points. $i=1,2 \ldots, n_{p}$ denote the number of piezoelectric actuators, $M_{p x}$ is the moment acting on the beam by the piezoelectric actuators and is given by

$$
M_{p x}^{i}(x, t)=K_{a} V_{a i}\left[H\left(x-x_{1 i}\right)-H\left(x-x_{2 i}\right)\right]
$$

where $V_{a i}$ is the voltage applied by the $i$ th actuator. $H($.$) is$ the step function. $x_{1 i}$ and $x_{2 i}$ denote the location of the two ends of the $i$ th piezoelectric actuator along the $X$ axis, and $K_{a}$ is a constant based on the properties of the beam and the piezoelectric actuators

$$
K_{a}=\frac{A_{p} e_{33} d_{p}}{h_{s t}}
$$

where $d_{p}$ is is the vertical distance from the neutral axis of the vehicle body to the vertical centre of the piezoelectric actuator. 
The end conditions for both free ends $\left(x=0\right.$ and $\left.x=L_{v}\right)$ of the beam are

$$
\begin{aligned}
& \left.\frac{\partial^{2} z(x, t)}{\partial x^{2}}\right|_{x=0}=\left.\frac{\partial^{3} z(x, t)}{\partial x^{3}}\right|_{x=0}=0 \\
& \left.\frac{\partial^{2} z(x, t)}{\partial x^{2}}\right|_{x=L_{v}}=\left.\frac{\partial^{3} z(x, t)}{\partial x^{3}}\right|_{x=L_{v}}=0
\end{aligned}
$$

By modal analysis technique, the solution of the transverse deflection $z(x, t)$ is assumed to be in the form

$$
z(x, t)=z_{3 c}(t)+\theta(t)\left(x-L_{b} / 2\right)+\sum_{r=1}^{\infty} q_{r}(t) \phi_{r}(x)
$$

where $q_{r}(t)$ is the $r$ th flexible mode in the generalized coordinate and $\phi_{r}(x)$ is the corresponding mode shape, which is determined from the eigenvalue problem

$$
\begin{aligned}
\phi_{r}(x)= & \cosh \lambda_{r} x+\cos \lambda_{r} x-\frac{\cosh \left(\lambda_{r} l\right)-\cos \left(\lambda_{r} l\right)}{\sinh \left(\lambda_{r} l\right)-\sin \left(\lambda_{r} l\right)} \\
& \left(\sinh \left(\lambda_{r} x\right)+\sin \left(\lambda_{r} x\right)\right)
\end{aligned}
$$

The frequency equation of an undamped free-free beam is

$$
\cos \left(\lambda_{r} l\right) \cosh \left(\lambda_{r} l\right)-1=0
$$

through which, a series of $\lambda_{r}$ can be determined, and the corresponding natural frequencies $\omega_{r}$ are determined by

$$
\lambda^{4}=\frac{\omega^{2} A_{b} \rho_{b}}{E I}
$$

Using solutions of Equations (13)-(15), the orthogonality properties of the mode shapes, and Dirac's delta function property

$$
\int_{-\infty}^{\infty} \delta^{(n)}(t-\theta) \phi(t) d t=(-1)^{n} \phi^{(n)}(\theta)
$$

where $\delta^{(n)}$ is the $n$th derivative of $\delta$ and $\phi$ is continuous at $\theta$. Uncoupled ordinary differential equations representing the flexible motions of the beam can be obtained by integrating equation (7) over the beam length

$$
\begin{gathered}
\ddot{q}_{r}(t)+2 \zeta_{r} \omega_{r} \dot{q}_{r}(t)+\omega_{r}^{2} q_{r}(t)=\frac{K_{a}}{M_{b}} \sum_{i=1}^{n_{p}} \Psi_{a r i} V_{a i}(t) \\
+\frac{F_{x_{L}} \phi_{r}\left(l_{1}\right)}{M_{b}}+\frac{F_{x_{R}} \phi_{r}\left(l_{2}\right)}{M_{b}}
\end{gathered}
$$

where structural damping is added and $\zeta_{r}$ is the damping ratio of the $r^{\text {th }}$ flexible mode, and $\Psi_{\text {ari }}$ is for the $r$ th mode and $i$ th piezoelectric actuator and is given by

$$
\Psi_{a r i}=\frac{d \phi_{r}}{d x}\left(x_{2 i}\right)-\frac{d \phi_{r}}{d x}\left(x_{1 i}\right)
$$

The first flexible mode has shown the most significant influence on ride quality of the railway vehicle $[2,17]$. For practical purposes only the first two flexible modes are considered in the model, and the corresponding mode shapes are shown in Figure 3. It is shown that each mode has its node points, i.e. where the corresponding mode shape is zero. The first flexible mode has its two node points near the positions of the suspension points and the second flexible mode has its node point at the middle of the beam. According to the forcing terms at the right hand side of Equation [17], the absolute value of a mode shape at certain point is an evaluation of importance of the control force acting at that point and the absolute value of $\Psi_{\text {ari }}$ at certain place is an evaluation of the importance of the the $i$ th piezoelectric actuator for the $r$ th mode.



Fig. 3: Mode shapes of the free-free beam of a railway vehicle

Piezoelectric laminar sensors are bonded to the structure for measuring the flexible modes. They produce voltage signals proportional to the average curvature. They are advantageous when only the vibrations of the flexible modes have to be measured and the signal part of the rigid body modes has to be eliminated [1]. The induced voltage $v_{p j}$ by the $j^{\text {th }}$ piezoelectric sensor is given by [6]

$$
v_{s p j}(t)=K_{s} \sum_{r=1}^{\infty} \Psi_{r j} q_{r}(t)
$$

where

$$
\Psi_{s r j}=\frac{d \phi_{r}}{d x}\left(x_{2 j}\right)-\frac{d \phi_{r}}{d x}\left(x_{1 j}\right)
$$

where $\Psi_{s r j}$ is for the $r$ th mode and $j$ th piezoelectric sensor, and $x_{1 j}$ and $x_{2 j}$ denote the position of the two ends of the $j$ th sensor. $K_{s}$ is related to the properties of the sensor and the beam.

Transform the description of the flexible model by Equation (17) to the state-space form as follows

$$
\begin{aligned}
\dot{x_{f}} & =\mathrm{A}_{\mathrm{f}} x_{f}+\mathbf{B}_{\mathrm{f} 1} u_{1}+\mathbf{B}_{\mathrm{f} 2} d \\
\boldsymbol{y}_{1} & =\mathrm{C}_{\mathrm{v}} x_{f}
\end{aligned}
$$

where the state vector $x_{f}$ is chosen as

$$
\boldsymbol{x}_{\boldsymbol{f}}(t)=\left[\begin{array}{llll}
\dot{q}_{1}(t) & q_{1}(t) & \dot{q}_{2}(t) & q_{2}(t)
\end{array}\right]
$$

$\boldsymbol{u}_{1}$ is the $\left(n_{p} \times 1\right)$ vector of voltage inputs to the piezoelectric actuators, $\boldsymbol{d}=\left[\begin{array}{ll}F_{x_{L}} & F_{x_{\boldsymbol{R}}}\end{array}\right]$ is the vector of force inputs from suspensions, $\boldsymbol{y}_{1}$ is the $\left(m_{p} \times 1\right)$ vector of measured variables by piezoelectric sensors, and input matrices and output ma- 
trices are as following

$$
\begin{aligned}
\mathbf{B}_{\mathbf{f} 1}= & \frac{K_{a}}{M_{v}}\left[\begin{array}{cccc}
\Psi_{a 11} & \Psi_{a 12} & \cdots & \Psi_{a 1 n_{\boldsymbol{p}}} \\
0 & 0 & \cdots & 0 \\
\Psi_{a 21} & \Psi_{a 22} & \cdots & \Psi_{a 2 n_{\boldsymbol{p}}} \\
0 & 0 & \cdots & 0
\end{array}\right] \\
\mathbf{B}_{\mathbf{f} \mathbf{2}}= & {\left[\begin{array}{cc}
\frac{\phi_{1}\left(x_{L}\right)}{M_{v}} & \frac{\phi_{1}\left(x_{\boldsymbol{R}}\right)}{M_{v}} \\
0 & 0 \\
\frac{\phi_{2}\left(x_{L}\right)}{M_{v}} & \frac{\phi_{2}\left(x_{\boldsymbol{R}}\right)}{M_{v}} \\
0 & 0
\end{array}\right] } \\
\mathbf{C}_{\mathbf{v}}= & \frac{K_{s}}{C_{p}}\left[\begin{array}{cccc}
0 & \Psi_{s 11} & 0 & \Psi_{s 21} \\
0 & \Psi_{s 12} & 0 & \Psi_{s 22} \\
0 & \vdots & 0 & \vdots \\
0 & \Psi_{s 1 m_{\boldsymbol{p}}} & 0 & \Psi_{s 2 m_{\boldsymbol{p}}}
\end{array}\right]
\end{aligned}
$$

\subsection{The overall model}

Combining the rigid model by Equation (3) and the flexible model by Equation (21), the overall model of the system in its state space form is given by

$$
\begin{aligned}
\dot{\boldsymbol{x}}= & {\left[\begin{array}{cc}
\mathbf{A}_{r}(12 \times 12) & \mathbf{A}_{\mathbf{f r}}(12 \times 4) \\
\mathbf{A}_{\mathbf{r f}}(4 \times 12) & \mathbf{A}_{\mathbf{f}}(4 \times 4)
\end{array}\right] \boldsymbol{x}+\left[\begin{array}{c}
\mathbf{B}_{\mathbf{r}} \\
\mathbf{B}_{\mathbf{f} \mathbf{1}}
\end{array}\right] \boldsymbol{u} } \\
& +\left[\begin{array}{c}
\mathbf{0} \\
\mathbf{B}_{\mathbf{f} \mathbf{2}}
\end{array}\right] \boldsymbol{V}_{a}+\left[\begin{array}{c}
\boldsymbol{G} \\
\mathbf{0}
\end{array}\right] \boldsymbol{w}
\end{aligned}
$$

where

$$
x=\left[\begin{array}{ll}
x_{r} & x_{f}
\end{array}\right]
$$

and $\mathbf{A}_{\text {fr }}$ and $\mathbf{A}_{\mathbf{r f}}$ are coupling matrices of the flexible and rigid states.

Based on the parameters given in [17], the bounce and pitch modes are at frequencies of $0.67 \mathrm{~Hz}$ and $0.85 \mathrm{~Hz}$ with damping ratios of 0.16 and 0.2 respectively and the first flexible mode is at $8.45 \mathrm{~Hz}$ and the second flexible mode is around $23.2 \mathrm{~Hz}$

\subsection{Placement of piezoelectric actuators and sensors}

$\mathcal{H}_{2}$ and $\mathcal{H}_{\infty}$ structural norms are used for evaluating the importance of actuators and sensors at their given positions. Here, we follow the approach in [3], where the norms of the $r^{\text {th }}$ mode can be approximately determined by

$$
\left\|G_{r}\right\|_{2} \cong \frac{\left\|B_{r}\right\|_{2}\left\|C_{r}\right\|_{2}}{2 \sqrt{\zeta_{r} \omega_{r}}} \quad\left\|G_{r}\right\|_{\infty} \cong \frac{\left\|B_{r}\right\|_{2}\left\|C_{r}\right\|_{2}}{2 \zeta_{r} \omega_{r}}
$$

And the $\mathcal{H}_{2}$ (or $\mathcal{H}_{\infty}$ ) norm of the $r^{\text {th }}$ mode with a set of actuators/sensors is the rms sum of $H_{2}\left(\right.$ or $\mathcal{H}_{\infty}$ ) norm of the mode with each single actuator/sensor.

$$
\left\|G_{r}\right\|_{2} \cong \sqrt{\sum_{i=1}^{n_{p}} \mid G_{r i} \|_{2}^{2}} \quad\left\|G_{r}\right\|_{\infty} \cong \sqrt{\sum_{i=1}^{n_{p}} \mid G_{r i} \|_{\infty}^{2}}
$$

According to the definition of the structural norms and the input and output matrices in equations (23) to (25), it is preferable to place the piezoelectric actuators and sensors at positions where $\Psi_{a r i}$ and $\Psi_{s r j}$ have high absolute values. As shown in Figure 4, the norms reach maxima at $\mathrm{x}=(13.5)$ for the first flexible mode and $x=7.84$ and $x=(19.16)$ for the second flexible mode.

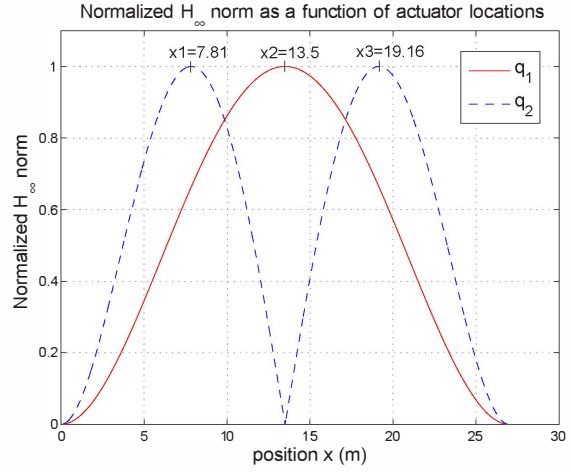

Fig. 4: Normalized $\mathcal{H}_{\infty}$ (or $\mathcal{H}_{2}$ ) indices as a function of actuator location with fixed sensor

Therefore, as it is shown by Figure 5, piezoelectric stack actuators (20 in parallel in order to achieve the necessary moments) and patch sensor are placed at $x=(13.5)$ for suppressing the $1^{\text {st }}$ flexible mode, and at $x=(7.84)$ and $x=(19.16)$ for suppressing the $2^{\text {nd }}$ flexible mode.

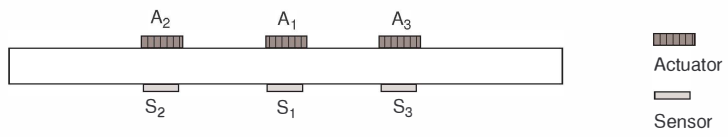

Fig. 5: Configuration of piezoelectric actuators and sensors

\section{Track profile and assessment method}

The assessment of ride quality relates to vehicle running on straight track with vertical misalignments (irregularities). For secondary suspension assessment, Gaussian white noise with a flat spectrum is a good approximation for the track velocity inputs [13]

$$
\dot{S}_{T}\left(\omega_{t}\right)=2 \pi \Omega_{v} v \quad\left(m s^{-1}\right)^{2}(\mathrm{rad} / \mathrm{s})^{-1}
$$

where $v$ is the velocity of the vehicle and $\Omega_{v}$ is the roughness factor, which is taken as $2.5 \times 10^{-7}$ for a good quality mainline track. A delay of $0.35 \mathrm{~s}$ between the front and rear track inputs is included.

Root mean square (RMS) values of vertical accelerations of the vehicle body are used to quantify the ride quality, in this case without frequency weighting. Frequency domain techniques are used to obtain RMS values and the power spectral densities (PSDs) of the signals of interest including body accelerations $\left(\ddot{z}_{x_{L}}, \ddot{z}_{x_{C}}\right.$ and $\left.\ddot{z}_{x_{R}}\right)$ and suspension deflections $\left(z_{3 L}-z_{1 L}\right.$ and $\left.z_{3 R}-z_{1 R}\right)$ in response to track irregularities.

\section{Controller design}

The controller design aims to reduce the vertical accelerations of the vehicle body while keeping the suspension deflections small. Hence a control law needs to be designed to determine the moments from the piezoelectric actuators and the forces from the active suspension actuators so that both the rigid mode and flexible mode vibration are suppressed.

Decentralize control strategy is adopted. Figure 6 shows the closed-loop system with structural damping controller 




Fig. 6: Structure of decentralized control

$K_{1}$ and suspension controller $K_{2} . G(s)$ represents the full model, $\boldsymbol{u}_{1}$ is piezoelectric actuator control input vector, $\boldsymbol{y}_{1}$ is measurements by the piezoelectric sensors, $\boldsymbol{u}_{2}$ is suspension control input vector, and $\boldsymbol{y}_{\mathbf{2}}$ is measurement output vector of accelerations of the vehicle body.

\subsection{Active structural damping with piezoelectric actua- tors and sensors}

Linear Quadratic Gaussian (LQG) control is applied for the design $K_{1}(s)$. It aims to minimize the accelerations of flexible motion against disturbances. The flexible model (21) is used for control design.

Performance positions are chosen as the centre of the vehicle, $x_{C}$, left suspension point, $x_{L}$, and right suspension point, $x_{L}$. Accelerations of the flexible modes at the above three positions are chosen as the controlled variables.

$$
\boldsymbol{y}_{\boldsymbol{f}}=\left[\begin{array}{c}
\phi_{1}\left(x_{C}\right) \ddot{q}_{1}+\phi_{2}\left(x_{C}\right) \ddot{q}_{2} \\
\phi_{1}\left(x_{L}\right) \ddot{q}_{1}+\phi_{2}\left(x_{L}\right) \ddot{q}_{2} \\
\phi_{1}\left(x_{R}\right) \ddot{q}_{1}+\phi_{2}\left(x_{R}\right) \ddot{q}_{2}
\end{array}\right]
$$

The problem is to devise a feedback-control law which minimizes the cost

$$
J=\lim _{T \rightarrow \infty} E\left\{\int_{0}^{T}\left[\boldsymbol{y}_{\boldsymbol{f}}(t)^{T} \mathbf{Q} \boldsymbol{y}_{\boldsymbol{f}}(t)+\boldsymbol{u}^{T}(t) \mathbf{R} \boldsymbol{u}(t)\right]\right\}
$$

where $\mathbf{R}_{\mathbf{0}}$ is the input weighting matrix and its elements are fixed at $1 . \mathrm{Q}_{\mathrm{y}}$ is the output weighting matrix and is chosen according to Bryson's rule [18]

$$
\mathbf{Q}_{\mathbf{y}}=\operatorname{diag}\left\{1 /\left(q_{y 1}\right)^{2}, 1 /\left(q_{y 2}\right)^{2}, 1 /\left(q_{y 3}\right)^{2}\right\}
$$

where $q_{y 1}, q_{y 2}$ and $q_{y 3}$ are initially given the expected values of the controlled variables and further tuned accordingly to achieve the best performance while maintaining the input voltages of the piezoelectric actuators within their limits.

Kalman filter is designed to estimate the states of the system that cannot be measured. The system is subject to disturbances from the secondary suspensions $d$. The variance of the disturbance is obtained from the simulation results of the passive system of the railway system. Piezoelectric sensor patches measure the strain in the beam by feeding back voltage signals. The measurement noise is assumed to have a root mean square value of one percent of the maximum value measured by the sensors, however, is further tuned until the estimator can provide a good performance for the system.

\subsection{Active suspension control}

Modal control with skyhook damping is adopted for active suspension control. Figure 7 shows the structure of the control system. Two linear accelerometers are used to measure vehicle body accelerations at the two suspension points, $\ddot{z}_{x_{L}}$ and $\ddot{z}_{x_{\boldsymbol{R}}}$. The measured signals are decomposed into modal components (i.e. bounce $\ddot{z}_{b}$ and pitch $\ddot{z}_{p}$ ). Then the bounce and pitch modes are controlled separately with skyhook damping, and finally the modal control signals are recombined and feed to the actuators.

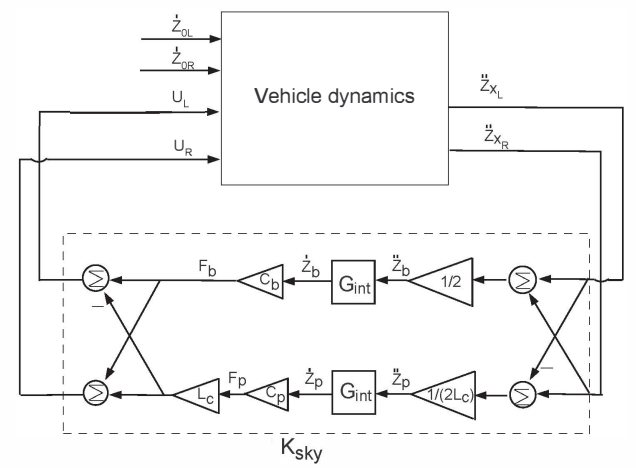

Fig. 7: Modal control with skyhook damping

Skyhook damping is a simple conventional method known to give sufficient improvements in ride quality of railway vehicles. The control action of an ideal skyhook damper is dependent upon the absolute velocity of a vehicle body.

$$
\begin{aligned}
& F_{b}=-c_{b} \dot{z}_{b} \\
& F_{c}=-c_{p} \dot{z}_{p}
\end{aligned}
$$

where $F_{b}$ and $F_{c}$ are the modal control forces, $c_{b}$ and $c_{p}$ are the modal skyhook damping gains, and $\dot{z}_{b}$ and $\dot{z}_{p}$ are the absolute velocity of the bounce and pitch modes of the vehicle body. In practice, the acceleration of the vehicle body is measured and then the signal is integrated to get the absolute velocity. A high pass filter is used along with an integrator in order to eliminate the long-term drift caused by offset effect of the transducer due to environmental reasons. A second order Butterworth high-pass filter (HPF) is used

$$
H_{h p}=\frac{s^{2} / \omega^{2}}{s^{2} / \omega^{2}+s 2 \zeta / \omega+1}
$$

where the cut-off frequency $\omega$ is chosen to be significantly less than the main suspension frequency, and in this case is chosen to be $0.1 \mathrm{~Hz}$.

\section{Simulation results and discussion}

The results are based on the actuator and sensor specifications given in Appendix and vehicle parameters given in [17].

Figure 8-10 compare the PSDs of systems $G+K_{1}, G+K_{2}$ and $G+K_{1}+K_{2}$ in response to track irregularities. It is shown that the first flexible mode (around $8 \mathrm{~Hz}$ ) is the main cause of degradation of ride quality while the second flexible mode (around $23 \mathrm{~Hz}$ ) is as small as negligible. With controller $K_{1}$, only the first flexible mode vibration is reduced. With controller $K_{2}$, the rigid mode vibrations at frequencies $<1 \mathrm{~Hz}$ are substantially reduced while the first flexible mode vibration is only slightly reduced. With combined controller $K_{1}+K_{2}$ both the rigid and flexible mode vibrations 
are reduced and the first flexible mode achieved a lower level of vibration than $K_{1}$.

Figure 11 compares suspension control forces for cases $G+K_{2}$ and $G+K_{1}+K_{2}$. Small reduction in the force inputs for $G+K_{1}+K 2$ around the flexible mode frequencies are observed. Figure 12 compares between voltage inputs of piezoelectric actuator A1 for $G+K_{1}$ and $G+K_{1}+K_{2}$. It is shown that, for $G+K_{1}+K_{2}$, smaller efforts are demanded from the piezoelectric actuators than $G+K_{1}$, and the piezoelectric control efforts become more concentrated around the flexible mode frequencies. This suggests that more efficient control from piezoelectric actuators are achieved with combined approach $K_{1}+K_{2}$.

Table 1 compares the RMS results between the passive system, closed-loop systems $G+K_{1}, G+K_{s k y}$ and $G+$ $K_{1}+K_{s k y}$ in response to vertical track irregularities. $G+K_{1}$ achieved substantial acceleration reduction at center of the vehicle $x_{c}$, while the accelerations at $x_{L}$ and $x_{R}$ are only slightly reduced. This is because the center acceleration is influenced the most by the first flexible modes. Significant reductions in all three accelerations are achieved by $G+K_{2}$ ,while the center acceleration is slightly higher than $G+K_{1}$. With $G+K_{1}+K_{2}$, a further reduction in center acceleration is brought by piezoelectric actuators compared to $G+K_{2}$. However, both the suspension control forces and piezoelectric actuator voltages are reduced compared to $G+K_{1}$ and $G+K_{2}$ respectively. This also suggests that the combined approach helps to use the piezoelectric actuators and suspension actuators more efficiently to address the overall vibration issues.

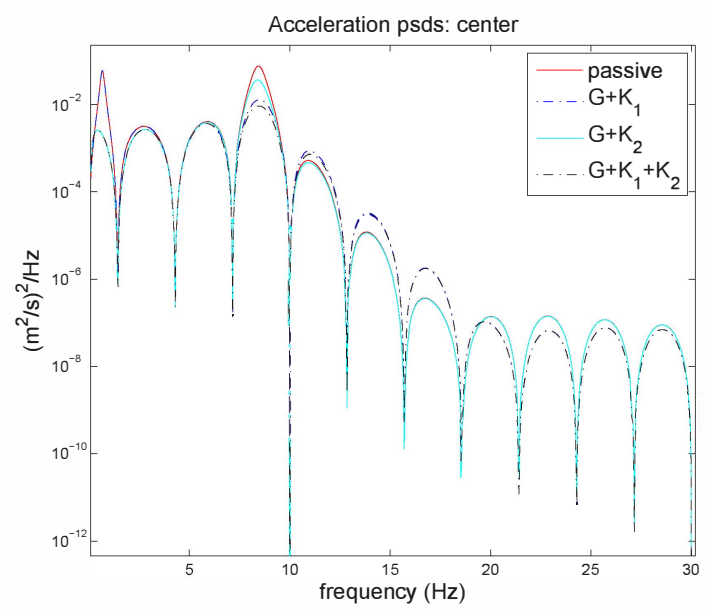

Fig. 8: Comparison between $G+K_{1}, G+K_{2}$ and $G+K_{1}+$ $K_{2}$ : PSDs of $\ddot{z}_{3 c}$

\section{Conclusion}

Combined active structural damping and active suspension control is investigated for vibration control of typical flexible-bodied railway vehicles. The design is based on a simplified side-view model of the railway vehicle considering only the first two flexible modes. It is shown that combined active structural damping and active suspension control work more efficiently in a complementary manner to each other that both the rigid and flexible mode vibrations are suppressed effectively.

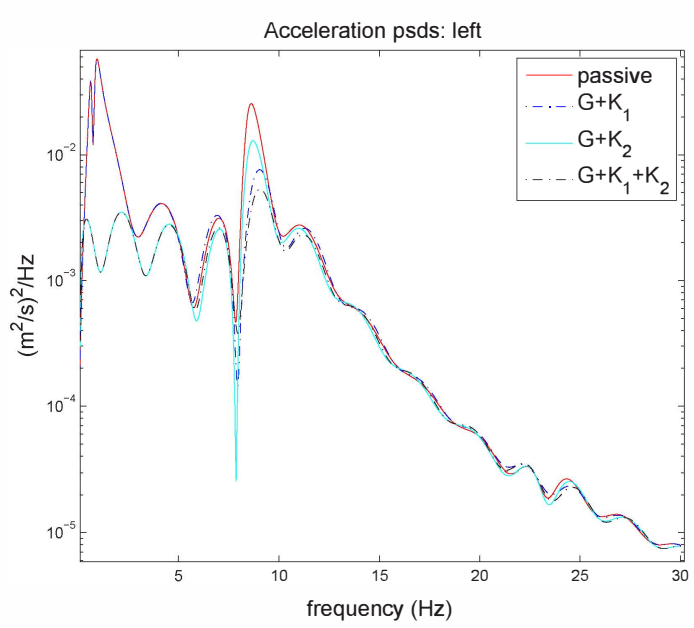

Fig. 9: comparison between $G+K_{1}, G+K_{2}$ and $G+K_{1}+$ $K_{2}$ : PSDs of $\ddot{z}_{3 l}$



Fig. 10: comparison between $G+K_{1}, G+K_{2}$ and $G+K_{1}+$ $K_{2}:$ PSDs of $\ddot{z}_{3 r}$


Fig. 11: comparison between $G+K_{2}$ and $G+K_{1}+K_{2}$ : PSDs of $U_{l}$ and $U_{r}$ 
Table 1: RMS results: comparison between $G+K_{1}, G+K_{2}$ and $G+K_{1}+K_{2}$

\begin{tabular}{|c|c|c|c|c|c|c|c|c|c|c|}
\hline \multirow{2}{*}{ System } & \multicolumn{3}{|c|}{$\begin{array}{c}\text { Vert. Acc. } \\
(\% g)\end{array}$} & \multicolumn{2}{c|}{$\begin{array}{c}\text { Susp. Def. } \\
(\mathrm{mm})\end{array}$} & $\begin{array}{c}\text { susp. forces } \\
(\mathrm{kN})\end{array}$ & \multicolumn{3}{c|}{$\begin{array}{c}\text { Piezo Act. Voltages } \\
(\text { volts })\end{array}$} \\
\cline { 2 - 14 } & centre & left & right & left & right & left & right & A1 & A2 & A3 \\
\hline$G$ & 3.04 & 3.04 & 3.75 & 7.8 & 11.35 & - & - & - & - & - \\
\hline$G+K_{1}$ & 2.15 & 2.79 & 3.5 & 7.80 & 11.35 & - & - & 289.3 & 192.6 & 191.3 \\
\hline$G+K_{2}$ & 2.19 & 1.76 & 1.83 & 11.09 & 6.76 & 3.52 & 2.56 & - & - & - \\
\hline$G+K_{1}+K_{2}$ & 1.56 & 1.73 & 1.72 & 9.81 & 7.75 & 2.95 & 2.59 & 242.3 & 160.57 & 160.86 \\
\hline
\end{tabular}

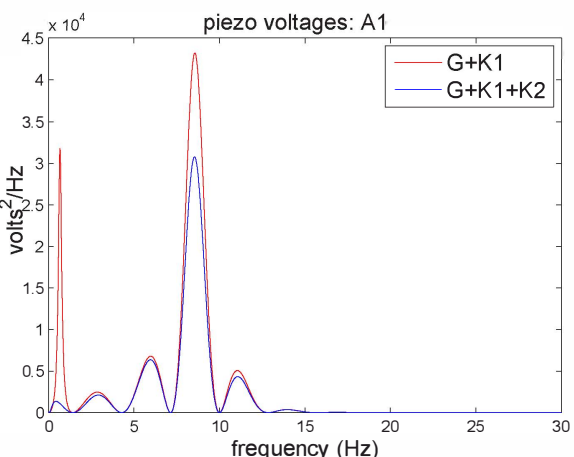

Fig. 12: comparison between $G+K_{1}$ and $G+K_{1}+K_{2}$ : PSDs of voltage to piezoelectric actuators A1

\section{References}

[1] Christian Benatzky, Martin Kozek, and Hanns Peter Jorgl. Comparison of controller design methods for a scaled metro vehicle - flexible structure experiments. In Proceedings of the 2007 American Control Conference, New York, USA, 2007.

[2] E. Foo and R.M. Goodall. Active suspension control of flexible-bodied railway vehicles using electro-hydraulic and electro-magnetic actuators. Control Engineering Practice, 8: 507-518, 2000.

[3] Wodek K. Gawronski. Advanced Structural Dynamics and Active Control of Structures. Springer, New York, 1998.

[4] Aleksander Hac, Iijoong Youn, and Hsien H. Chen. Control of suspensions for vehicles with flexible bodies-part I: Active suspensions. Journal of Dynamic Systems, Measurement, and Control, 118:509-517, 1996.

[5] Aleksander Hac, Iijoong Youn, and Hsien H. Chen. Control of suspensions for vehicles with flexible bodies-part II: Semiactive suspensions. Journal of Dynamic Systems, Measurement, and Control, 118:519-525, 1996.

[6] Dunant Halim and S.O. Reza Moheimani. Spatial resonant control of flexible structures - application to a piezoelectric laminate beam. IEEE transactions on control systems technology, 9(1), 2001.

[7] J. Hansson, M. Takano, T. Takigami, Tomioka, and Y.Suzuki. Vibration suppression of railway vehicle carbody with piezoelectric elements (a study by using a scale model). JSME International Journal Series C., 47(2), 2003.

[8] Takayoshi Kamada, Takayuki Tohtake, Tetsuro Aiba, and Masao Nagai. Active vibration control of the railway vehicles by smart structure concept. Proceedings of International Symposium on Speed-up and Service Technology for Railway and Maglev Systems, 2005.

[9] Takayoshi Kamada, Ryohei Kiuchi, and Masao Nagai. Suppression of railway vehicle vibration by shunt damping using stack type piezoelectric transducers. Vehicle System Dynamics, 46(Supplement), 2008.

[10] Takayoshi Kamada, Kazuyuki Hiraizumi, and Masao Nagai. Active vibration suppression of lightweight railway vehicle body by combined use of piezoelectric actuators and linear actuators. Vehicle System Dynamics, 48(Supplement):73-87, 2010.

[11] Martin Kozek, Christian Benatzky, Alexander Schirrer, and AntonStribersky. Vibration damping of a flexible car body structure using piezo-stack actuators. Control Engineering Practice,doi:10.1016/j.conengprac.2009.08.001, 2009.

[12] H. Md Yusof, R.M. Goodall, and R. Dixon. Control strategies for active secondary suspension actuators. In Proc 22nd Symposium on Dynamics of Vehicles on Roads and Tracks, Manchester, UK, 2011.

[13] I. Pratt. Active Suspensions Applied to Railway Trains. PhD thesis, Loughborough University, UK, 1996.

[14] Gerhard Schandl, Peter Lugner, Christian Benatzky, Martin Kozek, and Anton Stribersky. Comfort enhancement by an active vibration reduction system for a flexible railway car body. Vehicle System Dynamics, 45(9), 2007.

[15] A. Schirrer and M. Kozek. Co-simulation as effective method for flexible structure vibration control. 16th Mediterranean Conference on Control and Automation, Ajaccio, France, 2008.

[16] Tadao Takigami and Takahiro Tomioka. Bending vibration suppression of railway vehicle carbody with piezoelectric elements(experimental results of excitation tests with a commuter car). Journal of Mechanical Systems for Transportation and Logistics, 1(1), 2008.

[17] Xiang Zheng, Argyrios C. Zolotas, and Roger M. Goodal1. Modelling of flexible-bodied railway vehicles for vibration suppression. In Proceedings of International Conference on Systems Engineering, Coventry, UK, 2006.

[18] Argyrios C. Zolotas. Advanced control strategies for tilting trains. PhD thesis, Loughborough University, 2002.

\section{Appendix: Specifications of piezoelectric actuators and sensors}

Table 2: Specification of piezoelectric stack actuator

\begin{tabular}{ll}
\hline Maximum force & $50,000 \mathrm{~N}$ \\
Length, $L_{p x}$ & $0.194 \mathrm{~m}$ \\
Thickness of one disk & $5 \times 10^{-4} \mathrm{~m}$ \\
Diameter of disk & $0.045 \mathrm{~m}$ \\
Piezoelectric constant, $d_{33}$ & $6.80 \times 10^{-10} \mathrm{~m} /$ volts \\
Compliance, $s_{33}^{E}$ & $23 \times 10^{-12} \mathrm{~m}^{2} / \mathrm{N}$ \\
\hline
\end{tabular}

Table 3: Specification of piezoelectric sensor patch

\begin{tabular}{ll}
\hline Length & $0.02 \mathrm{~m}$ \\
Thickness & $2.5 \times 10^{-4} \mathrm{~m}$ \\
Width & $0.025 \mathrm{~m}$ \\
Young's Modulus, $E_{p}$ & $6.70 \times 10^{10} \mathrm{~N} / \mathrm{m}^{2}$ \\
Capacitance, $C_{p}$ & $1.05 \times 10^{-7} \mathrm{~F}$ \\
Electromechanical coupling factor, $k_{31}$ & 0.34 \\
Voltage constant, $g_{31}$ & $-1.15 \times 10^{-2} \mathrm{Vm} / \mathrm{N}$ \\
\hline
\end{tabular}

